\title{
The Research of Multi-Agent System Task Allocation Based on Auction
}

\author{
WU Qisheng ${ }^{1}$, ZHANG Rui ${ }^{1}$, HE Yunlai ${ }^{1}$, WANG Aimin ${ }^{1}$, JU Yongfeng ${ }^{1}$ \\ School of Electronic and Control Engineering Chang'an University, Xi'an, 710064 \\ qshwu@chd.edu.cn, 18710982056@163.com
}

\begin{abstract}
Multi-Agent System (MAS) can be used in emergency rescue in traffic monitor and aviation field ect.. It has been a hot spot about how to control the isomerous rescue robot team Multi-Agent System to finish the rescue task efficiently in the shortest time according to the task allocation based on the spot environment and existing resource now. In this thesis, a modified Multi-Agent task allocation algorithm based on auction is introduced, in the algorithm, robots act as agents to constitute the emergency rescue system, and it can realize the cooperation of Multi-Agent Robots to finish the rescue tasks with various of levels and kinds by the optimized task allocation.
\end{abstract}

Keywords: Multi-Agent System emergency rescue task allocation

\section{Introduction}

In recent years, Multi-Agent System (MAS) has become a hot spot in Distributed Artificial Intelligence (DIA), it has a vast potential for further development in the file of emergency rescue. For example, the environment changed quickly, and the aim-task is complicated, so multi-agent synergism can finish the task that single agent is hard to finish more quickly and efficiently [1]. To achieve multi-agent synergism rescue, the core problem to be solved is how to control isomerous rescue robot team Multi-Agent System to finish the rescue task effectively in the shortest time according to the task allocation based on the spot environment and existing resource. The way of task allocation mainly includes centralized model and decentralized model [2]. In centralized model, central agent decomposes and allocates the task according to present environment. In decentralized model, Gerkey designed a task allocation system, MURDOCH [3]. In the reference [4-5], Multi-Agent task allocation algorithm based on auction ( ABTA ) / ( ABGC ) was put forward to realize the allocation of task, this algorithm used the principle that the agent who find task auction. But by this way, the agents need to patrolling in the spot all the time. In the reference [6], the optimal allocation based on the contact net was used, in this way of allocation, Manager agent is in charge of estimating the task, decomposing the task into multiple subtask, and surveilling the situation of task accomplishment. Bidder agent is in charge of bidding and finishing the task. But in this way of task allocation, Manager agent needs to allocate subtask according to the priority. In the serious fire spot, this way of task allocation can't realize finishing the whole task quickly. As the Multi-Agent rescue system is a mixed system including centralized model and decentralized model [7], in this thesis, centralized model and decentralized model are combined, using Modified Multi-Agent task allocation algorithm based on auction to allocate the task, according to gather information of fire spot by the sensors, the wireless nodes deliver the information to the control center. If the control center inspects the occurring of fire, it will transmit the rescue agents to put it out.

\section{Algorithm improvement, system design, experiment and data}

Multi-Agent task allocation algorithm based on auction is introduced detailedly in reference [4-6].To make sure the system gets the maximal profit and pays minimum cost, it must satisfy (1), $E$ is the profit; $C$ is the cost .

$$
\max \sum_{j=0}^{m} \sum_{i=0}^{n}(E-C)
$$

In this thesis, the Modified Multi-Agent task allocation algorithm based on auction is used, and applied to the emergency rescue. The task is the fire, and the agents are rescue robots. The auction agent is setting as the ControlCenter, it is invariable, and the information of the spot is inspected by the sensors and delivered by the wireless nodes. The Control-Center makes judgement on the situation of the spot. If the Control-Center finds the fire, that is the aim-task generates, it will announce that the auction begins; every rescue robot receives the estimation of the fire from the Control-Center before deciding whether to bid or not based on the condition itself; after the Control-Center obtains the bid information of rescue robots, it deals with the bid, chooses the suitable robots and teams, that is that the Control-Center has already chosen the suitable rescue robot team, at last the Control-Center publishes the bid information to every rescue robot, it realized the combination between the centralized model and decentralized model in Multi-Agent System.

Fire $T_{j} \quad$ includes three eigenvalues, $T_{j}=\left\{T_{j}\right.$ Kind,$T_{j}$ Level,$T_{j}$ State $\} . T_{j}$ Kind is the kind of the fire $T_{j} ; T_{j}$ Level is the level of the fire $T_{j} ; T_{j}$ State is the state of the fire $T_{j}$, so there are three matrixes :

$$
\begin{aligned}
& \text { TKind }=\left(\begin{array}{llll}
T_{1} \text { Kind } & T_{2} \text { Kind } \cdots & T_{j} \text { Kind } \cdots & T_{n} \text { Kind }
\end{array}\right) \\
& \text { TLevel }=\left(\begin{array}{llll}
T_{1} \text { Level } & T_{2} \text { Level } \cdots T_{j} \text { Level } & \cdots & T_{n} \text { Level }
\end{array}\right)
\end{aligned}
$$


TState $=\left(T_{1}\right.$ State $\quad T_{2}$ State $\cdots \quad T_{j}$ State $\cdots \quad T_{n}$ State $)$

Based on the "building fire-extinguisher configuration design specification" that puts into effect in October.2005, there are five kinds of fire:A,B,C,DandE, its sorts and way of putting out are introduced in detail in reference[8].So

$T_{j}$ Kind $=\{\mathrm{A}, \mathrm{B}, \mathrm{C}, \mathrm{D}, \mathrm{E}\} ; T_{j}$ Level $=\{1,2,3\}$, and the level is higher, the fire is more serious; $T_{j}$ State $=\{0,1\}$, and 0 shows the fire put out successfully, 1 shows failed.

Agent $A_{j}$ includes three eigenvalues,

$A_{i}=\left\{A_{i}\right.$ Kind,$A_{i}$ Level, $A_{i}$ State $\} . A_{j}$ Kind shows the kind of the rescue robots $A_{j} ; A_{j}$ Level shows the level of the rescue robots $A_{j} ; A_{j}$ State shows the state of the rescue robots $A_{j}$, so there are three matrixes :

$$
\begin{aligned}
& \text { AKind }=\left(\begin{array}{llllll}
A_{1} \text { Kind } & A_{2} \text { Kind } & \cdots & A_{i} \text { Kind } & \cdots & A_{m} \text { Kind }
\end{array}\right)^{\mathrm{T}} \\
& \text { ALevel }=\left(\begin{array}{llllll}
A_{1} \text { Level } & A_{2} \text { Level } & \cdots & A_{i} \text { Level } & \cdots & A_{m} \text { Level }
\end{array}\right)^{\mathrm{T}} \\
& \text { AState }=\left(\begin{array}{llllll}
A_{1} \text { State } & A_{2} \text { State } & \cdots & A_{i} \text { State } & \cdots & A_{m} \text { State }
\end{array}\right)^{\mathrm{T}}
\end{aligned}
$$

Corresponding to $T_{j}$ Kind,

$A_{i}$ Kind $=\{\mathrm{A} \backslash \mathrm{B}, \mathrm{B} \backslash \mathrm{C}, \mathrm{C} \backslash \mathrm{E}, \mathrm{D}\} ; A_{i} L$ evel $=\{1,2\}$, and the level is higher, the fire-extinguisher that the rescue robot equipped is more; $A_{i}$ State $=\{0,1\}$, and 0 shows the rescue robot $A_{j}$ is busy, 1 shows it is not busy.

$P$ shows the property of the system in the present task, $P$ includes two eigenvalues , $P=\{$ PKind, PLeve $\}$, PKind shows the property kind of the system in the present task, PLevel shows the level property of the system in the present task, so there are two matrixes :

$$
\text { PKind }=\left(\begin{array}{ccccc}
\text { PKind }_{11} & \cdots & \text { PKind }_{1 j} & \cdots & \text { PKind }_{1 n} \\
\vdots & \ddots & \vdots & \ddots & \vdots \\
\text { PKind }_{i 1} & \cdots & \text { PKind }_{i j} & \cdots & \text { PKind }_{i n} \\
\vdots & \ddots & \vdots & \ddots & \vdots \\
\text { PKind }_{m 1} & \cdots & \text { PKind }_{m j} & \cdots & \text { PKind }_{m n}
\end{array}\right)
$$$$
\text { PLevel }\left(\begin{array}{ccccc}
\text { PLevel }_{11} & \cdots & \text { PLevel }_{1 j} & \cdots & \text { PLevel }_{1 n} \\
\vdots & \ddots & \vdots & \ddots & \vdots \\
\text { PLevel }_{i 1} & \cdots & \text { PLevel }_{i j} & \cdots & \text { PLevel }_{i n} \\
\vdots & \ddots & \vdots & \ddots & \vdots \\
\text { PLevel }_{m 1} & \cdots & \text { PLevel }_{m j} & \cdots & \text { PLevel }_{m n}
\end{array}\right)
$$

Through PKind, PLevel and given algorithm, the result matrix $R$ can be gotten, in this matrix, the Control-Center can get all the combinations of the rescue robot teams based on the bid information gotten. TKind, TLevel, TState are generated automatically by the Control-Center based on the information getting; the way of calculating PKind, PLevel, $R$ are stated as follows :

Definition I : If the kind of the fire matches with the kind of the rescue robot, the product of corresponding element will be 1 , if not, it will be 0 .
PKind $=$ AKind $*$ TKind $=$

$\left(\begin{array}{ccccc}\text { PKind }_{11} & \cdots & \text { PKind }_{1 j} & \cdots & \text { PKind }_{1 n} \\ \vdots & \ddots & \vdots & \ddots & \vdots \\ \text { PKind }_{i 1} & \cdots & \text { PKind }_{i j} & \cdots & \text { PKind }_{i n} \\ \vdots & \ddots & \vdots & \ddots & \vdots \\ \text { PKind }_{m 1} & \cdots & \text { PKind }_{m j} & \cdots & \text { PKind }_{m n}\end{array}\right)$

Definition II : Supposing there is an $\mathrm{m}^{*} \mathrm{n}$ matrix $A$, defining $1 / A$ is an $\mathrm{m}^{*} \mathrm{n}$ matrix too, its element is the reciprocal of the corresponding element of the matrix $A$, that is:

$A=\left(\begin{array}{ccc}a_{11} & \ldots & a_{1 n} \\ \vdots & \ddots & \vdots \\ a_{m 1} & \cdots & a_{m n}\end{array}\right) 1 / A=\left(\begin{array}{ccc}1 / a_{11} & \ldots & 1 / a_{1 n} \\ \vdots & \ddots & \vdots \\ 1 / a_{m 1} & \cdots & 1 / a_{m n}\end{array}\right)$

PLevel $=$ ALevel $^{*}(1 /$ TLevel $)=$

$\left(\begin{array}{ccccc}\text { PLevel }_{11} & \cdots & \text { PLevel }_{1 j} & \cdots & \text { PLevel }_{1 n} \\ \vdots & \ddots & \vdots & \ddots & \vdots \\ \text { PLevel }_{i 1} & \cdots & \text { PLevel }_{i j} & \cdots & \text { PLevel }_{i n} \\ \vdots & \ddots & \vdots & \ddots & \vdots \\ \text { PLevel }_{m 1} & \cdots & \text { PLevel }_{m j} & \cdots & \text { PLevel }_{m n}\end{array}\right)$

Definition III: Supposing there are two matrixes that have the same type $A$ and $B$, the coupled product of matrix $A$ and $B$ is record as $C=A \odot B$, specifying $C_{i j}=A_{i j} * B_{i j}$, that is:

$A \odot B=\left(\begin{array}{ccccc}a_{11} * b_{11} & \cdots & a_{i j} * b_{1 j} & \cdots & a_{1 n} * b_{1 n} \\ \vdots & \ddots & \vdots & \ddots & \vdots \\ a_{i 1} * b_{i 1} & \cdots & a_{i j} * b_{i j} & \cdots & a_{i n} * b_{i n} \\ \vdots & \ddots & \vdots & \ddots & \vdots \\ a_{m 1} * b_{m 1} & \cdots & a_{m j} * b_{m j} & \cdots & a_{m n} * b_{m n}\end{array}\right)$

$R=$ PKind $\odot$ PLeve $=$

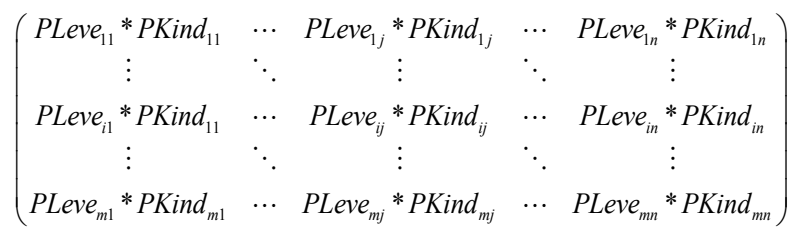

The Control-Center can get all the possible combinations of the rescue robot team based on the result matrix $R$, its way of calculating states as follows:

Definition IV: Supposing there is an $\mathrm{m}^{*} \mathrm{n}$ matrix $A$, defining $i A(0 \leq i \leq m)$ is an $\mathrm{m}^{*} 1$ matrix, its elements is the $i$ row elements of the matrix $A$,that is:

$A=\left(\begin{array}{ccccc}a_{11} & \cdots & a_{1 i} & \cdots & a_{1 n} \\ \vdots & \ddots & \vdots & \ddots & \vdots \\ a_{m 1} & \cdots & a_{m i} & \cdots & a_{m n}\end{array}\right) \quad i A=\left(\begin{array}{c}a_{1 i} \\ \vdots \\ a_{m i}\end{array}\right)$

For fire $T_{1}, T_{1}$ Result $=$ AState $\odot 1 R$, that is: 
$T_{1}$ Result $=$ AState $\odot 1 R=$

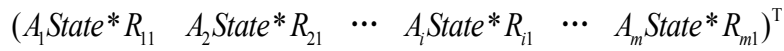

For $T_{1}$ Result, the rescue robot whose line subscript is nonzero can be chosen. If $0<T_{1}$ Result $_{i}<1$, the level of the robot is incapable of the level of the fire, in this situation, multiple robots should be chosen, and the sum of the corresponding elements in $T_{1}$ Result should greater than 1 ; If $T_{1}$ Result $_{i}=1$, the level of the robot is just capable of the level of the fire, in this situation, the corresponding robot could be chosen; If $T_{1}$ Result $_{i}>1$, the level of the robot is capable of the level of the fire, in this situation, the corresponding robots could be chosen, but doing this may cause waste.

Ruled in algorithm, after choosing the rescue robot team for the fire $T_{1}$, the Control-Center needs updating the data of the AState before choosing the rescue robot team for the fire $T_{2}$, until the Control-Center has chosen the rescue robot teams for all the fires, and a kind of combination can be gotten, the Control-Center calculates $\sum_{j=0}^{m} \sum_{i=0}^{n}(E-C)$, and saves the result. Then the Control-Center calculates other combinations of the rescue robot team again, and repeats the process of abovementioned until the combination is the same as the combination that has gotten. Finally the Control-Center publishes the result of the auction based on the principle of (1), the rescue robots that win the bid sign the contract with the Control-Center, and the auction is over.

In experiment design, there are 8 rescue robots in the system, 2 of them equipped with foam extinguisher, and the level is 1 and $2 ; 2$ of them equipped with carbon dioxide extinguisher, and the level is 1 and 2; 2 of them equipped with portable ammonium phosphate extinguisher, and the level is 1 and 2; the last equipped with metal dedicated extinguisher, and the level is 1 and 2. Supposing there are 4 fires in the spot, 2 of them are caused by solid matter, and the level is 1 and 2; 1 of them is caused by mental matter, and the level is 3; the last is caused by matter burning with electricity, and the level is 1 . So the matrixes TKind, TLeve, TState, AKind, ALeve, AState, PKind, PLevel and $R$ are:

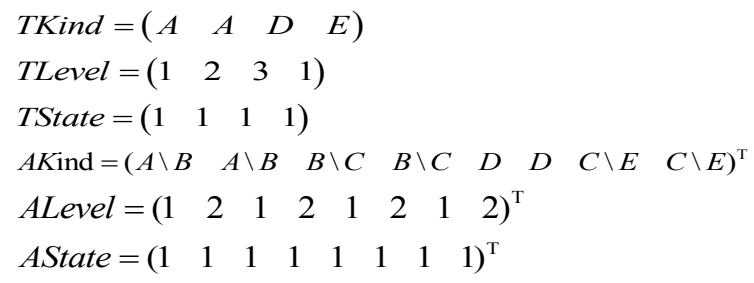

$$
\begin{aligned}
& {\text { PLevel }=\text { ALevel }^{*}(1 / \text { TLevel })}=\left(\begin{array}{cccc}
1 & 1 / 2 & 1 / 3 & 1 \\
2 & 1 & 2 / 3 & 2 \\
1 & 1 / 2 & 1 / 3 & 1 \\
2 & 1 & 2 / 3 & 2 \\
1 & 1 / 2 & 1 / 3 & 1 \\
2 & 1 & 2 / 3 & 2 \\
1 & 1 / 2 & 1 / 3 & 1 \\
2 & 1 & 2 / 3 & 2
\end{array}\right) \\
& R=\text { PKind } \square \text { PLevel }=\left(\begin{array}{cccc}
1 & 1 / 2 & 0 & 0 \\
2 & 1 & 0 & 0 \\
0 & 0 & 0 & 0 \\
0 & 0 & 0 & 0 \\
0 & 0 & 1 / 3 & 0 \\
0 & 0 & 2 / 3 & 0 \\
0 & 0 & 0 & 1 \\
0 & 0 & 0 & 2
\end{array}\right)
\end{aligned}
$$

\section{Analysis}

Matrixes $T_{1}$ Result,$T_{2}$ Result,$T_{3}$ Result,$T_{4}$ Result can be gotten based on matrix $R$, so the first combination of the rescue robot team can be gotten :

$T_{1}$ Result $=$ AState $\odot 1 R=\left(\begin{array}{llllllll}1 & 2 & 0 & 0 & 0 & 0 & 0 & 0\end{array}\right)^{\mathrm{T}}$

For the fire $T_{1}$, the rescue robot $A_{1}$ can be transmitted, and the matrix AState is:

AState $=\left(\begin{array}{llllllll}0 & 1 & 1 & 1 & 1 & 1 & 1 & 1\end{array}\right)^{\mathrm{T}}$

$T_{2}$ Result $=$ AState $\odot 2 R=\left(\begin{array}{llllllll}0 & 1 & 0 & 0 & 0 & 0 & 0 & 0\end{array}\right)^{\mathrm{T}}$

For the fire $T_{2}$, the rescue robot $A_{2}$ can be transmitted, and the matrix AState is:

AState $=\left(\begin{array}{llllllll}0 & 0 & 1 & 1 & 1 & 1 & 1 & 1\end{array}\right)^{\mathrm{T}}$

$T_{3}$ Result $=$ AState $\odot 3 R=\left(\begin{array}{llllllll}0 & 0 & 0 & 0 & 1 / 3 & 2 / 3 & 0 & 0\end{array}\right)^{\mathrm{T}}$

For the fire $T_{3}$, the rescue robots $A_{5}$ and $A_{6}$ can be transmitted, and the matrix AState is:

$$
\begin{aligned}
& \text { AState }=\left(\begin{array}{lllllllll}
0 & 0 & 1 & 1 & 0 & 0 & 1 & 1
\end{array}\right)^{\mathrm{T}} \\
& T_{4} \text { Result }=\text { AState } \odot 4 R=\left(\begin{array}{llllllll}
0 & 0 & 0 & 0 & 0 & 0 & 1 & 2
\end{array}\right)^{\mathrm{T}}
\end{aligned}
$$

For the fire $T_{4}$, the rescue robot $A_{7}$ can be transmitted, and the matrix $E$ and $C$ are:

$$
E=\left(\begin{array}{llll}
1 & 0 & 0 & 0 \\
0 & 2 & 0 & 0 \\
0 & 0 & 0 & 0 \\
0 & 0 & 0 & 0 \\
0 & 0 & 1 & 0 \\
0 & 0 & 2 & 0 \\
0 & 0 & 0 & 1 \\
0 & 0 & 0 & 0
\end{array}\right) C=\left(\begin{array}{llll}
1 & 0 & 0 & 0 \\
0 & 2 & 0 & 0 \\
0 & 0 & 0 & 0 \\
0 & 0 & 0 & 0 \\
0 & 0 & 1 & 0 \\
0 & 0 & 2 & 0 \\
0 & 0 & 0 & 1 \\
0 & 0 & 0 & 0
\end{array}\right)
$$

The Control-Center calculates $\sum_{j=0}^{4} \sum_{i=0}^{8}(E-C)=0$ in this combination of the rescue robot team, by the same way, the 
Control-Center calculates other combinations of the rescue robot team and its $\sum_{j=0}^{m} \sum_{i=0}^{n}(E-C)$.

So the final result calculated by the Control-Center is: for the fire $T_{1}, A_{1}$ wins the bid; for the fire $T_{2}, A_{2}$ wins the bid; for the fire $T_{3}, A_{5}$ and $A_{6}$ win the bid; for the fire $T_{4}, A_{7}$ wins the bid.

\section{Conclusion}

In the algorithm, when different kinds of and levels of fires occur in the spot, the rescue robot team transmitted by the Control-Center can put out the fire efficiently and successfully. And it has been verified by the project.

The disadvantage of the research is that the kind and quantity of the combustible near the fires isn't considered, so the Control-Center can't forecast the tendency of the fires in the further to put out the fires more quickly and efficiently.

\section{Acknowledgement}

Thanks for the funding and support of follow projects: Major science and technology Project of The 12th Five-Year Plan for national economic and social development of the People's Republic of China (20111318812260); Central University special funds for basic research and Operating Expense Project of Chang'an University (Innovation Team CHD2011TD018); Henan zhongyuan high-speed technology project based on content networking of highway passenger vehicle and special vehicle operation environmental monitoring management system; Department of science and technology of Shaanxi Province Project Research on the key technology of WSN and image based on underground station fire perception (2011K06-31) ; Chang'an University 2012 national college innovation project (201210710048).

\section{Reference}

[1] VANG Yintao, YAN Weisheng, YAN Wei. Application of Multi-agent Consensus to Robot Formation Control [J]: China: College of Marine, Northwestern Polytechnic University, Xi'an 710071. 2010.35 (10):4-7.

[2] Gerkey P.Mataric J. A Formal Analysis and Taxonomy of Task Allocation in Multi-robot Systems [J]: International Journal of Robotics Research. 2004.23 (3):939-954.

[3] Gerkey B P. On multi-agent task allocation [D]: [Eng. D thesis]. USA: University of Southern California, 2003

[4] YU Wentao. The Research and Application of Multi-Agent System Task Allocation [D]: [M. E. thesis]. Chain: Central South University. 2008

[5] HONG Chanhao. Research on Multiple Agent Rescue Simulation System [D]: [M .E .thesis]. China: Harbin Engineering University. 2011

[6] LU Xiaohui. Research of Decision-making of Platoon Agent in RoboCupRescue Simulation System [D]: [M. E. thesis].China: Guangzhou Industrial University. 2012

[7] ZHANG Xiaoyong. Research on Planning and Adaptive Collaborating in RoboCupRescue Simulation System [D]: [Eng. D thesis]. Chain: Central South University.2009

[8] Building Fire-Extinguisher Configuration Design Specification [GB 50140-2005]. 